ARTIGO

https: / / doi.org/10.22239/2317-269x.01518

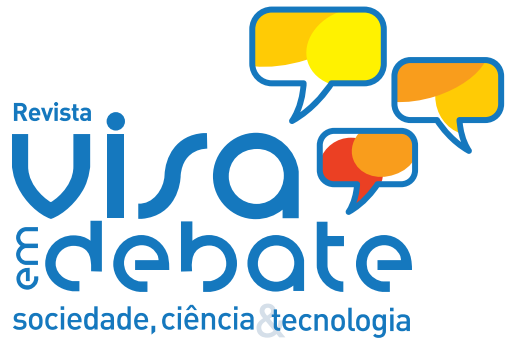

sociedade, ciência tecnologia

\title{
Principais irregularidades em estabelecimentos sujeitos a controle sanitário
}

\author{
Main irregularities in establishments subject to sanitary control
}

\section{Daniela Fernandes César* (iD \\ Patrícia Maria de Faria e Silva (ID \\ Saraid da Costa Figueiredo (D) \\ Filipe Curzio Laguardia (iD}

\begin{abstract}
RESUMO
Introdução: A fiscalização sanitária verifica o cumprimento de normas e regulamentos técnicos visando segurança e qualidade. A Vigilância Sanitária de Minas Gerais possui um sistema de notificação de situações de risco sanitário que possibilita a coleta sistemática e análise de dados relativos às inspeções sanitárias. Esses dados são usados para identificação do risco sanitário no território e consequente definição de ações. Objetivo: Determinar as irregularidades mais frequentemente encontradas durante as inspeções sanitárias realizadas em Minas Gerais. Método: Estudo transversal das irregularidades sanitárias declaradas por fiscais sanitários de Minas Gerais nos formulários relativos às inspeções, inseridas na Planilha de Notificações de Riscos e Situações de Riscos 2019. As frequências encontradas de cada tipo de irregularidade foram determinadas e ordenadas. Resultados: As irregularidades mais comumente encontradas, decrescentemente, são: problemas de Documentação/ Garantia da qualidade; infraestrutura inadequada; irregularidades de Organização e Higiene; irregularidades em equipamentos, incluindo manutenção preventiva e calibração; falhas na qualidade dos produtos; recursos humanos; e problemas de gerenciamento de resíduos. Todas essas irregularidades têm graus variados de potencial risco sanitário, mesmo que estejam, muitas vezes, sujeitas às mesmas medidas, educativas e/ou coercitivas, por parte da Vigilância Sanitária. Conclusões: 0 conhecimento das principais irregularidades encontradas nos estabelecimentos inspecionados é uma informação de alta relevância para a fiscalização sanitária para um diagnóstico territorial, otimização do processo de trabalho e para orientar as ações de educação e instrução, de forma a permitir uma efetiva intervenção dos serviços nesses problemas.
\end{abstract}

PALAVRAS-CHAVE: Vigilância Sanitária; Inspeção Sanitária; Risco Sanitário
Secretaria de Estado de Saúde de Minas Gerais. Belo Horizonte, MG, Brasil

\section{* E-mail: daniela.cesar@saude.mg.gov.br}

Recebido: 01 abr 2020 Aprovado: 06 nov 2020

\begin{abstract}
Introduction: Sanitary inspection verifies compliance with technical standards and regulations for safety and quality. The Health Surveillance of Minas Gerais has a notification procedure for sanitary risk situations that allows the systematic collection and analysis of data related to health inspections. These data are used to identify the sanitary hazard in the territory and the consequent definition of actions. Objective: To determine the irregularities most frequently encountered during health inspections carried out in Minas Gerais. Method: Cross-sectional study of sanitary irregularities reported by health inspectors of Minas Gerais in the form of risk notification and risk situation sheet 2019 (Planilha de Notificações de Riscos e Situações de Riscos 2019). The frequencies found for each type of irregularity were determined and ordered. Results: The most commonly found irregularities are, in decreasing order: Documentation/Quality Assurance issues; Inadequate infrastructure; Organization and Hygiene irregularities; Equipment irregularities, including preventive maintenance and calibration; Failure in product quality; Human resources, and waste management issues. All of these irregularities have varying degrees of potential health risk, even though they are often subject to the same course of action, educational and/or coercive, by the Health Surveillance. Conclusions:
\end{abstract}


The knowledge of the main irregularities found in the inspected establishments is a highly relevant information for health inspection for the purposes of territorial diagnosis, optimization of the work process and to guide the education and instruction actions, in order to allow an effective intervention of the services in these problems.

KEYWORDS: Health Surveillance; Health Inspection; Health Risk

\section{INTRODUÇÃO}

As práticas de vigilância sanitária no Brasil tiveram seu início amalgamado às necessidades médicas da população, numa época em que o país ainda era colônia de Portugal'. No decorrer dos anos foram editadas outras normas que ajudariam a conferir à vigilância sanitária as características que ainda hoje a definem, notadamente a Lei $n^{\circ} 6.437$, de 20 de agosto de $1977^{2}$, que define o que configura infração à legislação sanitária federal e estabelece as sanções respectivas, além de estabelecer os ritos do processo administrativo-sanitário e a chamada Lei do Sistema Único de Saúde (SUS), Lei $n^{\circ} 8.080$, de 19 de setembro de $1990^{3}$, que dispõe sobre as condições para a promoção, proteção e recuperação da saúde, a organização e o funcionamento dos serviços correspondentes e dá outras providências, de onde se cunhou o mais conhecido conceito de vigilância sanitária:

um conjunto de ações capaz de eliminar, diminuir ou prevenir riscos à saúde e de intervir nos problemas sanitários decorrentes do meio ambiente, da produção e circulação de bens e da prestação de serviços de interesse da saúde, abrangendo: o controle de bens de consumo que, direta ou indiretamente, se relacionem com a saúde, compreendidas todas as etapas e processos, da produção ao consumo; e o controle da prestação de serviços que se relacionam direta ou indiretamente com a saúde.

Dentre essas ações estão as de controle, como licenciamento, fiscalização sanitária e monitoramento, que visam a verificação da aplicação das normas pelos estabelecimentos ${ }^{4}$. A forma que os estabelecimentos se valem para se adequarem à conformidade exigida numa inspeção sanitária é conhecida como Boas Práticas. A Agência Nacional de Vigilância Sanitária (Anvisa), criada em 1999 pela Lei ${ }^{\circ} 9.782$, de 26 de janeiro ${ }^{5}$, conceitua Boas Práticas de Fabricação (BPF), como um conjunto de procedimentos estabelecidos que relacionam práticas produtivas cuidadosamente criadas e revisadas, que se estendem desde o desenvolvimento dos produtos e a compra de insumos e componentes, passando pelo processo produtivo, armazenamento, até a comercialização dos produtos, incluindo a vigilância da manutenção dos requisitos de qualidade quando de posse do consumidor (vigilância pós-mercado) ${ }^{6}$ - conceito cuja essência não difere das empregadas em outros tipos de estabelecimentos (Boas Práticas Clínicas, Boas Práticas de Manipulação de Medicamentos, Boas Práticas de Manipulação de Alimentos e as demais).

De fato, as Boas Práticas são normas de procedimentos estabelecidas com o objetivo de atingir um determinado padrão de identidade e qualidade de um produto e/ou serviço que consideram, de maneira geral, quatro pontos principais a serem analisados: pontos críticos de controle e práticas de pessoal; instalações; requisitos gerais de equipamentos; e controles de produção ${ }^{7}$. Essa amplitude de ação, assim como seu objetivo, faz com que a observância (ou não) das Boas Práticas guarde estreita relação com as infrações sanitárias. Isso se dá porque a ação da vigilância sanitária fundamenta-se nos mesmos riscos e ameaças de agravo que esses procedimentos intentam suprimir nos processos ${ }^{8}$. Uma evidência disso é o fato de que muitos dos regulamentos técnicos carregam no título a menção das Boas Práticas, como o Regulamento Técnico de Procedimentos Operacionais Padronizados aplicados aos Estabelecimentos Produtores/Industrializadores de Alimentos, a Lista de Verificação das Boas Práticas de Fabricação em Estabelecimentos Produtores/Industrializadores de Alimentos (Resolução da Diretoria Colegiada - RDC n²75, de 21 de outubro de $2002^{9}$ ), as Diretrizes Gerais de Boas Práticas de Fabricação de Medicamentos (RDC $\mathrm{n}^{\circ} 301$, de 21 de agosto de 201910), os Requisitos de Boas Práticas de Funcionamento para os Serviços de Diálise (RDC ${ }^{\circ} 11$, de 13 de março de 2014¹1), entre outros.

A fiscalização sanitária verifica a aplicação das Boas Práticas na forma do cumprimento de normas e regulamentos técnicos e indução à adoção de aprimoramentos com vista à melhoria da segurança e qualidade dos serviços prestados, dos processos produtivos e dos produtos de interesse da saúde ${ }^{4}$.

Com o avanço da descentralização das ações de vigilância sanitária no estado de Minas Gerais (MG), o órgão estadual de Vigilância Sanitária (Visa) implantou, para fins de acompanhamento das ações realizadas pelas Visa municipais, um instrumento cuja aplicação permite a identificação do risco sanitário no território e a definição de ações voltadas à mitigação/eliminação dos fatores de risco encontrados ${ }^{12}$.

Notificações de riscos e situações de risco da Vigilância Sanitária de Minas Gerais

A Superintendência de Visa da Secretaria de Saúde de MG, por meio do Grupo Técnico Vigi-Risco, criou em 2012 um formulário online para notificação de risco sanitário encontrado em inspeções, preenchido pelos fiscais sanitários municipais e estaduais ${ }^{13}$. Esse formulário, denominado Planilha de Notificações de Riscos e Situações de Riscos, foi abrigado na plataforma FormSus, ferramenta para criação de formulários no âmbito do DATASUS desenvolvida para dar agilidade, estruturação e qualidade ao processo de coletar e disseminar dados pela internet ${ }^{14}$.

O formulário inicialmente era de preenchimento voluntário, mas em 2016 a edição da deliberação da Comissão Intergestores 
Bipartite (CIB) - SUS/MG $\mathrm{n}^{\circ} 2.418$, de 17 de novembro ${ }^{15}$, tornou o preenchimento compulsório, fazendo dele um dos indicadores no Programa de Monitoramento das Ações de Vigilância em Saúde (indicador 21).

Por força da deliberação, os fiscais/autoridades sanitárias que exercem suas atividades no estado de MG, após a realização de cada inspeção, nos diferentes tipos de estabelecimentos sujeitos a fiscalização sanitária, devem preencher o mencionado formulário. A extensão do formulário é dependente de características do estabelecimento e das condições encontradas durante a inspeção - sendo sua menor versão composta de 18 questões e em sua totalidade de 163 questões - e a grande maioria dessas questões é objetiva.

As questões são divididas entre dados de identificação do ente fiscalizador e do estabelecimento; caracterização do estabelecimento conforme as três áreas da vigilância sanitária: alimentos, serviços de saúde e de interesse da saúde, e medicamentos e congêneres; situação encontrada na verificação do emprego das Boas Práticas e abordagens realizadas.

Apesar de guardar características de um questionário, a ferramenta é sempre mencionada como formulário, e tem por característica versões anuais para aprimoramento, com um link de acesso distinto. No momento do acesso inicial, são apresentadas 16 perguntas, passíveis de desdobramentos conforme as respostas preenchidas. Como parte dessas perguntas é específica a alguns tipos de estabelecimentos, dificilmente o total de perguntas (163) é preenchido em uma mesma inspeção.

Embora o formulário conte com estas perguntas específicas para alguns tipos de estabelecimento, tomando por base regulamentos técnicos da Anvisa ou do estado de MG, é importante ressaltar que esse formulário é um recurso padrão da mencionada superintendência, e, em que pese a riqueza de dados, o fato de abarcar diferentes estabelecimentos nivela esses estabelecimentos entre si. Na prática, os dados gerados serão representativos de suas classes, mas não permitem visualizações detalhadas de cada estabelecimento ou diferenciação destes por características particulares, como porte, tipos de processos, arranjos administrativos etc.

Após preenchimento das questões específicas, são preenchidas as questões sobre ações de intervenção proposta e/ou realizada. Entre estas está a pergunta se durante a inspeção foram encontradas irregularidades no estabelecimento inspecionado. Caso o fiscal declare ter sido encontrada irregularidade, uma questão que visa especificar os tipos de irregularidades encontrados é adicionada ao formulário. Nessa questão objetiva, as não conformidades foram categorizadas pelo Grupo Técnico Vigi-Risco em sete tipos de irregularidades possíveis, acrescidas da opção "Outro". A opção "Outro" apresenta adicionalmente um campo aberto para digitação para os casos em que o fiscal julgue que nenhuma das opções pré-definidas se aproxima da irregularidade encontrada.

Em cada opção de tipo de irregularidade, há exemplificação de não conformidades relacionadas, para facilitar a classificação por parte do responsável pelo preenchimento, conforme o Quadro.

Importante notar que essas opções só se tornam disponíveis uma vez declarada a irregularidade, mas não há máximo de opções a se marcar. Pode-se marcar qualquer número de opções, sendo essa a variável colhida e analisada por este estudo.

Quadro. Categorias das irregularidades sanitárias e exemplos de não conformidades relacionadas, conforme apresentadas nas opções da pergunta “Qual o tipo de irregularidade encontrada?” da Planilha de Notificações de Riscos e Situações de Riscos, uma vez que seja declarada pelo fiscal a presença de irregularidade na inspeção sanitária.

\begin{tabular}{|c|c|}
\hline Tipo de irregularidade & Exemplos de não conformidades relacionadas a cada tipo de irregularidade \\
\hline Estrutura física & $\begin{array}{l}\text { Irregularidades em pisos, tetos, paredes ou outra estrutura; problemas de ventilação e/ou iluminação; } \\
\text { estrutura incompatível com projeto arquitetônico aprovado; ausência/não aprovação de projeto } \\
\text { arquitetônico (quando exigível); dentre outros. }\end{array}$ \\
\hline Organização/Higiene & $\begin{array}{l}\text { Desorganização geral ou de algum ambiente; presença de sujidades; presença de objetos em desuso ou } \\
\text { incompatíveis com a atividade; dentre outros. }\end{array}$ \\
\hline Qualidade dos produtos & $\begin{array}{c}\text { Produtos vencidos; produtos não identificados ou identificados de forma incorreta; produtos armazenados } \\
\text { de forma incorreta; dentre outros. }\end{array}$ \\
\hline Documentação/Garantia da Qualidade & $\begin{array}{c}\text { Ausência de Alvará Sanitário; Alvará Sanitário vencido; ausência de documentos/certificados como alvará } \\
\text { de localização, certificado de dedetização, registro de limpeza de caixa d'água, manual de normas e } \\
\text { rotinas/POP, PGRSS; dentre outros. }\end{array}$ \\
\hline Recursos humanos & $\begin{array}{l}\text { Ausência de EPI; EPI incompleto ou inadequado à atividade; ausência de registros de capacitação, } \\
\text { imunização e/ou exames periódicos (se necessários); dentre outros. }\end{array}$ \\
\hline $\begin{array}{l}\text { Equipamentos, incluindo manutenção } \\
\text { preventiva e calibração }\end{array}$ & $\begin{array}{l}\text { Ausência de equipamentos exigidos para a atividade ou para os ambientes (geladeiras, autoclaves, } \\
\text { acessórios para higiene das mãos, extintores de incêndio etc.); equipamentos em mau funcionamento; } \\
\text { ausência de manutenção preventiva/corretiva; ausência de calibração e/ou registros de validação (se } \\
\text { necessários); dentre outros. }\end{array}$ \\
\hline Gerenciamento de resíduos & $\begin{array}{c}\text { Descarte incorreto de produtos; ausência ou número reduzido de lixeiras/recipientes adequados ao tipo } \\
\text { de resíduo descartado; lixeiras/recipientes dispostos em locais inadequados; ausência de implementação } \\
\text { do PGRSS; dentre outros. }\end{array}$ \\
\hline Outro & Apenas irregularidades que não pertencem a nenhuma das categorias acima, descritas de forma objetiva. \\
\hline
\end{tabular}

Fonte: Elaborado pelos autores, 2020.

POP: procedimento operacional padrão; PGRSS: Plano de Gerenciamento de Resíduos de Serviços de Saúde; EPI: equipamentos de proteção individual. 
A implementação dessas notificações possibilitou a criação de uma série histórica de dados, insumo fundamental para o monitoramento das situações de risco. Além disso, elas instruem o indicador 21 do Programa de Monitoramento das Ações de Vigilância em Saúde já mencionado. Atende, também, o inciso I do Art. 17 da Lei no 13.317, de 24 de setembro de 1999 (Código de Saúde do Estado de Minas Gerais) ${ }^{16}$, ao possibilitar a consolidação, análise e interpretação de dados indispensáveis relacionados à saúde.

A partir dos dados obtidos na Planilha de Notificações de Riscos e Situações de Risco 2019, que contém a totalidade dos formulários das inspeções sanitárias realizadas no estado de Minas Gerais e declaradas no ano de 2019, o presente trabalho teve como objetivo determinar as irregularidades mais comumente encontradas e, mediante a comunicação dos resultados, permitir que a intervenção da Visa para a sua regularização seja mais eficaz.

\section{MÉTODO}

Trata-se de um estudo transversal que tomou como fonte de dados os 159.325 registros feitos por fiscais sanitários municipais e estaduais na Planilha de Notificações de Riscos e Situações e Riscos. O período escolhido foi $1^{\circ}$ de fevereiro de 2019 a 31 de janeiro de 2020, correspondendo àquele em que os registros inseridos contemplam o tipo de irregularidade encontrada durante a inspeção. A inserção dessa qualificação do tipo de irregularidade só passou a figurar no formulário em 2019, o que ensejou a restrição no período considerado para esse levantamento. Essa informação possibilitou categorizar irregularidades e calcular as respectivas frequências. Esse recorte reduziu o universo de análise a 100.156 formulários. Em razão dos critérios estabelecidos pela organização do trabalho de cada serviço de Visa, não é possível fazer inferências relativas ao percentual de estabelecimentos inspecionados, nos termos do indicador 21 do Programa de Monitoramento das Ações de Vigilância em Saúde ${ }^{15}$. Em outras palavras, não há correspondência entre o número de formulários e o de estabelecimentos existentes, posto que o mesmo estabelecimento pode ter sido inspecionado mais de uma vez.

\section{RESULTADOS E DISCUSSÃO}

O banco de dados recebido continha 159.325 notificações. Foram selecionadas aquelas que declaravam ter sido encontrada alguma irregularidade, um total de 100.156 (62,86\%). Entretanto, no registro dos dados, verificou-se que a despeito da declaração de ter sido encontrada irregularidade, dois dos registros não discriminavam o tipo de irregularidade encontrada, sendo igualmente eliminados.

O objeto final foram 100.154 notificações que, além de declarar a existência de irregularidades, informaram de que tipo de irregularidade se tratava.

A inspeção sanitária de rotina visa determinar a adequação ou não de um estabelecimento sob controle sanitário aos requisitos legais, mas nem sempre apresenta o nível de conformidade à legislação ou traz o julgamento crítico dos itens analisados ${ }^{17}$.

Sem avaliação da criticidade dos requisitos, a análise dos indicadores, apenas em sua dicotomia conforme/não conforme, não revela o risco real associado a eles. Viterbo et al. ${ }^{17}$ definiram ainda a existência de risco potencial e risco clássico, tal que o risco clássico inclui probabilidades e o potencial relaciona-se a eventos em curso e seus possíveis efeitos. 0 risco potencial é o mais comumente tratado pelas inspeções sanitárias, e relacionar as inadequações encontradas - que configuram efetivamente o risco determinado - por suas frequências e associações mais comuns, permite o acompanhamento e a comparação de diversos objetos sob controle da vigilância sanitária.

Apesar dessa limitação, a análise das irregularidades declaradas se torna mais relevante quando não se restringe aos dados de apenas um estabelecimento, mas se estende tomando toda a informação disponível gerada durante as inspeções sanitárias por permitir melhores avaliações do potencial risco.

No levantamento realizado nesse estudo, a distribuição de inadequações se deu conforme quantitativo da Tabela. É importante destacar que existe a possibilidade de mais de um tipo de

Tabela. Quantitativo de respostas relativas às irregularidades encontradas e seu tipo, conforme declarado na Planilha de Notificações de Riscos e Situações de Riscos 2019, em ordem decrescente de ocorrência.

\begin{tabular}{|c|c|c|}
\hline Tipos de irregularidades & $\mathbf{N}^{\circ}$ & \%* \\
\hline Documentação/Garantia de Qualidade & 66.190 & $66,09 \%$ \\
\hline Estrutura Física & 45.610 & $45,54 \%$ \\
\hline Organização/Higiene & 29.158 & $29,11 \%$ \\
\hline Equipamentos, incluindo manutenção preventiva e calibração & 22.507 & $22,47 \%$ \\
\hline Qualidade dos produtos & 16.258 & $16,23 \%$ \\
\hline Recursos humanos & 14.128 & $14,11 \%$ \\
\hline Gerenciamento de resíduos & 15.858 & $13,84 \%$ \\
\hline Outros & 6.103 & $6,09 \%$ \\
\hline Total de notificações & 100.154 & $100,00 \%$ \\
\hline
\end{tabular}

Fonte: Elaborada pelos autores, 2020.

*Os tipos de irregularidades são variáveis independentes, devendo-se avaliar as frequências relativas apenas em relação ao total de registros, não havendo parâmetro mínimo ou máximo de ocorrências. 
irregularidade ser declarada em um mesmo registro, e por esse motivo as porcentagens encontradas mostram a relação desse quesito apenas com o total de registros e não com os demais requisitos, já que a soma dessas ocorrências, em porcentagens, ultrapassariam os $100,00 \%$.

A irregularidade mais comumente encontrada é aquela relativa à Documentação/Garantia da qualidade. Essa classe é exemplificada: em ausência de alvará sanitário; alvará sanitário vencido; ausência de documentos/certificados como alvará de localização, certificado de dedetização, registro de limpeza de caixa d'água, manual de normas e rotinas/Procedimento Operacional Padrão (POP), Plano de Gerenciamento de Resíduos de Serviços de Saúde (PGRSS); dentre outras. Em estudo de Barbosa e Costa ${ }^{18}$, o exame de documentos é o instrumento mais utilizado no processo de operacionalização de segurança sanitária. Em parte, a grande frequência de inadequação desse requisito pode ser explicada pela concentração de atenção nesse instrumento, e a subutilização de outros disponíveis, como o monitoramento de indicadores e aqueles baseados em eventos adversos (farmacovigilância e tecnovigilância, por exemplo) ${ }^{18,19}$.

A tendência de basear-se em documentação é histórica no desenvolvimento da vigilância sanitária. Explica Lucchese ${ }^{20}$ que a regulação mormente federal (como ocorria entre as décadas de 1940 e 1970) tinha intervenção eminentemente normativa pois era preciso regulamentar as indústrias e seus produtos. 0 componente fiscalizatório não foi estruturado com eficiência e o conhecimento técnico acabou sendo intensamente cartorial - baseado apenas em documentos. A perpetuação desse modelo, tal que o fiscal concentre sua atenção à documentação disponível, contribui e possivelmente explica o fato de ser essa a irregularidade mais declarada, embora na atualidade a avaliação da qualidade do sistema passe pela compreensão de que a prática anterior, apegada aos aspectos cartoriais, burocráticos ou estruturais não garante resultados e não atende à sociedade, sendo necessária uma nova forma de avaliação ${ }^{21}$.

O segundo tipo de irregularidade mais encontrada foi aquele relacionado aos requisitos de infraestrutura, no formulário exemplificado por: irregularidades em pisos, tetos, paredes ou outra estrutura; problemas de ventilação e/ou iluminação; estrutura incompatível com projeto arquitetônico aprovado; ausência/não aprovação de projeto arquitetônico (quando exigível); dentre outras.

As mudanças estruturais em geral demandam injeção de recursos e podem exigir um longo período de tempo, sendo possível que sua inadequação seja de um tipo cuja solução seja de mais longo prazo. Embora as falhas de infraestrutura possam comprometer a qualidade do serviço ou produto e colocar em risco o usuário, consumidor e os trabalhadores ${ }^{22}$, é importante ponderar sua frequência também à luz dos aspectos de execução da inspeção.

Giunta e Lacerda ${ }^{23}$, em um estudo sobre inspeções sanitárias em hospitais, relacionaram capacitação técnica com a avaliação da organização e com a execução de serviços específicos. Afirmaram ainda que os aspectos estruturais foram os mais citados à inspeção, o que pode indicar uma relação em que, sendo os aspectos estruturais mais conhecidos dos fiscais, é possível que a inadequação destes seja mais facilmente identificável. É sabido que um planejamento que leve em conta as atividades a serem desenvolvidas no dimensionamento e especificações das estruturas evita o aparecimento de problemas funcionais e estéticos ${ }^{24}$.

No formulário, as irregularidades de Organização/Higiene são exemplificadas por: desorganização geral ou de algum ambiente; presença de sujidades; presença de objetos em desuso ou incompatíveis com a atividade; dentre outras, sendo a terceira opção em frequência de respostas. A determinação de um fluxo de trabalho é geralmente estabelecida por um responsável que detenha conhecimento do processo produtivo e impactada positivamente pela sua presença no estabelecimento ${ }^{25}$. É uma inferência possível que o desconhecimento desses processos limite a avaliação das inadequações pelos fiscais, já que a grande gama de atividades econômicas sujeitas ao controle sanitário dificulta o conhecimento necessário a uma avaliação dos riscos associados aos respectivos processos produtivos.

O quarto grupo mais comum são as irregularidades em equipamentos, incluindo manutenção preventiva e calibração, definida por ausência de equipamentos exigidos para a atividade ou para os ambientes (geladeiras, autoclaves, acessórios para higiene das mãos, extintores de incêndio etc.); equipamentos em mau funcionamento; ausência de manutenção preventiva/corretiva; ausência de calibração e/ou registros de validação (se necessários); dentre outros. De acordo com Monteiro e Lessa ${ }^{26}$, a adaptação dos equipamentos e de seus fabricantes aos regulamentos técnicos estimula melhorias qualitativas nos produtos e processos de fabricação.

Sobre o quinto tipo mais comum de irregularidade, a qualidade dos produtos, deve ser destacado o impacto sobre a saúde dos potenciais consumidores. Há que se considerar que os produtos, uma vez disponíveis no mercado, estarão sujeitos também ao crivo daqueles que os consumirão. 0 mesmo vale para os serviços ${ }^{27}$. Nesse sentido, ao atender às denúncias, questionamentos e reclamações da população em relação a produtos e serviços, a Visa dá uma resposta adequada aos reais problemas sanitários ${ }^{28}$.

Dentre as irregularidades elencadas, as três menos declaradas foram as relativas: 1) recursos humanos, exemplificada por ausência de EPI; EPI incompleto ou inadequado à atividade; ausência de registros de capacitação, imunização ou exames periódicos, dentre outras; 2 ) gerenciamento de resíduos, incluindo o descarte incorreto; ausência/número reduzido/disposição em local inadequado de lixeiras ou recipientes; ausência de implementação do PGRSS; e 3) outros, que o próprio fiscal poderia incluir e que não é intenção desse estudo pormenorizar.

Por seu caráter, uma infração sanitária de qualquer natureza tem potencial de trazer risco sanitário à população, estando, portanto, sujeitas às mesmas medidas por parte da Visa (tanto educativas como coercitivas), mas a identificação da frequência com que cada um de seus tipos ocorre possibilita melhor detalhamento 
dos riscos e consequentemente melhor planejamento de intervenções por parte dos serviços sanitários no intento de salvaguardar a saúde da população, inclusive do ponto de vista da saúde dos trabalhadores, e a proteção ao meio ambiente ${ }^{29,30}$.

O conhecimento pela Visa das falhas gerenciais, estruturais e processuais mais comuns pode facilitar o planejamento do serviço, otimizar o processo de trabalho e orientar as atividades de capacitação da equipe técnica.

\section{CONCLUSÕES}

O presente estudo mostrou que $66,09 \%$ das irregularidades citadas pelos fiscais são aquelas da categoria Documentação/Garantia da Qualidade. Embora a bibliografia consultada explique esse achado, esperava-se que a maior frequência estivesse entre as categorias mais afetas ao processo produtivo, locus do risco sanitário que a ação da vigilância sanitária deve eliminar ou minimizar.
Essa constatação dá elementos para uma nova perspectiva no planejamento de capacitações aos fiscais, que podem ter mais facilidade na identificação de irregularidades documentais e de garantia de qualidade.

Faz-se necessário a continuidade desse estudo para identificação dos motivos pelos quais as irregularidades relacionadas à Documentação/Garantia da Qualidade ocorrem. Pode-se especular que parte dessas não conformidades tem origem na falta de clareza das exigências necessárias para o exercício das atividades sujeitas ao controle sanitário, sendo necessário ao setor público o desenvolvimento de ações educativas que visem mitigar esses problemas.

Quanto às demais irregularidades encontradas pelos fiscais sanitários, a determinação de suas frequências é uma informação de igualmente alta relevância, principalmente para o diagnóstico e planejamento de ações.

\section{REFERÊNCIAS}

1. Porto A, Sanglard G, Fonseca MRF, Costa RGR, organizadores. História da saúde no Rio de Janeiro: instituições e patrimônio arquitetônico (1808-1958). Rio de Janeiro: Fundação Oswaldo Cruz; 2008[acesso 2 mar 2020]. Disponível em: http://books.scielo.org/id/7f83x/epub/ porto-9788575415993.epub

2. Brasil. Lei $\mathrm{N}^{\circ} 6.437$, de 20 de agosto de 1977 . Configura infrações à legislação sanitária federal, estabelece as sanções respectivas, e dá outras providências. Diário Oficial União. 24 ago 1977.

3. Brasil. Lei $\mathrm{N}^{\circ} 8.080$, de 19 de setembro de 1990. Dispõe sobre as condições para a promoção, proteção e recuperação da saúde, a organização e o funcionamento dos serviços correspondentes e dá outras providências. Diário Oficial União. 20 set 1990.

4. Conselho Nacional de Secretários de Saúde - CONASS. Para entender a gestão do SUS volume 6, tomo 2: vigilância em saúde. Brasília: Conselho Nacional de Secretários de Saúde; 2007.

5. Brasil. Lei $N^{\circ}$ 9.782, de 26 de janeiro de 1999. Define o sistema nacional de vigilância sanitária, cria a agência nacional de vigilância sanitária, e dá outras providências. Diário Oficial União. 27 jan 1999.

6. Agência Nacional de Vigilância Sanitária - Anvisa. Guia de auxílio na implantação de boas práticas em produtos para saúde. Brasília: Agência Nacional de Vigilância Sanitária; 2020[acesso 5 set 2020]. Disponível em: http://portal.anvisa. gov.br/documents/219201/219401/ Guia\%2Bde\%2Baux\%25C3\%25ADlio\%2B\%25C3\%25A0s\%2BBPF pdf/051235af-82b8-46f9-94a5-b5921acd8611?version=1.0

7. Akutsu RC, Assunção BR, Barbosa CE, Oliveira SKE, Coelho AWC. Adequação das boas práticas de fabricação em serviços de alimentação. Rev Nutr. 2005;18(3):419-27. https://doi.org/10.1590/S1415-52732005000300013
8. Prá ML. A possibilidade jurídica do emprego de medidas coercitivas atípicas em processos administrativos sancionadores: aplicação específica em ações de vigilância sanitária [monografia]. Florianópolis: Universidade Federal de Santa Catarina; 2019.

9. Agência Nacional de Vigilância Sanitária - Anvisa. Resolução RDC $\mathrm{N}^{\circ} 275$, de 21 de outubro de 2002. Dispõe sobre o regulamento técnico de procedimentos operacionais padronizados aplicados aos estabelecimentos produtores/industrializadores de alimentos e a lista de verificação das boas práticas de fabricação em estabelecimentos produtores/industrializadores de alimentos. Diário Oficial União. 6 nov 2002.

10. Agência Nacional de Vigilância Sanitária - Anvisa. Resolução RDC No 301, de 21 de agosto de 2019. Dispõe sobre as diretrizes gerais de boas práticas de fabricação de medicamentos. Diário Oficial União. 22 ago 2019.

11. Agência Nacional de Vigilância Sanitária - Anvisa. Resolução RDC N ${ }^{\circ} 11$, de 13 de março de 2014. Dispõe sobre os requisitos de boas práticas de funcionamento para os serviços de diálise e dá outras providências. Diário Oficial União. 14 mar 2014.

12. Secretaria de Estado de Saúde de Minas Gerais - SES-MG. Relatório vigi-risco 2019. Belo Horizonte: Superintendência de Vigilância Sanitária; 2020.

13. Coelho AR, Cesar DF, Vieira LA, Silva PMF. Projeto vigi-risco: manual para preenchimento da planilha de notificações de riscos e situações de riscos: 2020 do FormSUS. Belo Horizonte: Secretaria do Estado de Saúde de Minas Gerais; 2020[acesso 29 mar 2020]. Disponível em: http://vigilancia. saude.mg.gov.br/index.php/download/manual-parapreenchimento-da-planilha-de-notificacoes-de-riscos-esituacoes-de-riscos-2018-do-formsus $/$ ?wpdmdl=4403

14. Ministério da Saúde (BR). FormSUS: manual do gestor. Brasília: Ministério da Saúde; 2015[acesso 21 mar 2020]. Disponível em: http://formsus.datasus.gov.br/manual/ formsus_manual.pdf 
15. Secretaria de Estado de Saúde de Minas Gerais - SES-MG. Deliberação $N^{\circ} 2.418$, de 17 de novembro de 2006. Aprova as normas gerais para participação, execução, acompanhamento, monitoramento e avaliação do programa de monitoramento das ações de vigilância em saúde, no âmbito do estado de Minas Gerais. Diário Oficial Estado. 18 nov 2016.

16. Governo do Estado de Minas Gerais. Lei $N^{\circ} 13.317$, de 24 de setembro de 1999. Contém o código de saúde do estado de Minas Gerais. Diário Oficial do Estado. 25 set 1999.

17. Viterbo LMF, Dinis MAP, Sá KN, Marques CASDC, Navarro MVT, Leite HJD. Desenvolvimento de um instrumento quantitativo para inspeção sanitária em serviços de alimentação e nutrição, Brasil. Cienc Saude Coletiva. 2020;25(3):805-16. https://doi.org/10.1590/1413-81232020253.16372018

18. Barbosa AO, Costa EA. Os sentidos de segurança sanitária no discurso da agência nacional de vigilância sanitária. Cienc Saude Coletiva. 2010;15(Supl.3):3361-70. https://doi.org/10.1590/S1413-81232010000900011

19. Souza GS, Costa EA. Considerações teóricas e conceituais acerca do trabalho em vigilância sanitária, campo específico do trabalho em saúde. Cienc Saude Coletiva. 2010;15(Supl.3):3329-40. https://doi.org/10.1590/S1413-81232010000900008

20. Lucchese G. Descentralização e modelo sistêmico: o caso da vigilância sanitária. Cienc Saude Coletiva. 2010;15(Supl.3):3318-28. https://doi.org/10.1590/S1413-81232010000900003

21. Eduardo MBP. Vigilância sanitária: para gestores municipais de serviços de saúde. São Paulo: Universidade de São Paulo; 1998[acesso 15 jul 2019]. Disponível em: http://bvsms.saude. gov.br/bvs/publicacoes/saude_cidadania_volume08.pdf

22. Freitas F, Oliveira SB. Irregularidades sanitárias como marcador de risco à saúde: um desafio para a vigilância sanitária. Vigil Sanit Debate. 2013;1(1):43-51. https://doi.org/10.3395/vd.v1i1.4

23. Giunta APN, Lacerda RA. Inspeção dos programas de controle de infecção hospitalar dos serviços de saúde pela vigilância sanitária: diagnóstico de situação. Rev Esc Enferm USP. 2006;40(1):64-70. https://doi.org/10.1590/S0080-62342006000100009

24. Rolim RIE, Basso C. Estrutura física de restaurantes universitários do Rio Grande do Sul. Discip Sci Ser Cienc Biol Saude. 2016;17(1):83-94.

25. Akutsu RC, Botelho RA, Camargo EB, Sávio KEO, Araújo WC. Adequação das boas práticas de fabricação em serviços de alimentação. Rev Nutr. 2005;18(3):419-27. https://doi.org/10.1590/S1415-52732005000300013

26. Monteiro EC, Lessa ML. A metrologia na área de saúde: garantia da segurança e da qualidade dos equipamentos eletromédicos. Engevista. 2005;7(2).

27. Behrens I, Chociai JG. A cosmetovigilância como instrumento para a garantia da qualidade na indústria de produtos cosméticos. Visao Acad. 2007;8(1):31-5. https://doi.org/10.5380/acd.v8i1.11663

28. Fonseca SGO, Fonseca EP. Ações de vigilância sanitária no município de Divinópolis, Minas Gerais, entre 2008 e 2013. Vigil Sanit Debate. 2014;2(1):27-32. https://doi.org/10.3395/vd.v2i1.59

29. Silva NFC, Vianna CMM, Oliveira FSG, Mosegui GBG, Rodrigues MPS. Fuzzy visa: um modelo de lógica fuzzy para a avaliação de risco da vigilância sanitária para inspeção de resíduos de serviços de saúde. Physis. 2017;27(1):127-46. https://doi.org/10.1590/s0103-73312017000100007

30. Assi M. Gestão de riscos com controles internos: ferramentas, certificações e métodos para garantir a eficiência dos negócios. São Paulo: Saint Paul; 2018.

Contribuição dos Autores

César DF, Silva PMF - Concepção, planejamento (desenho do estudo), aquisição, análise, interpretação dos dados e redação do trabalho. Figueiredo SC - Aquisição, análise e interpretação dos dados e redação do trabalho. - Aquisição, análise dados e redação do trabalho. Laguardia FC- Planejamento (desenho do estudo) e redação do trabalho. Todos os autores aprovaram a versão final do trabalho.

Os autores informam não haver qualquer potencial conflito de interesse com pares e instituições, políticos ou financeiros deste estudo.

Esta publicação está sob a licença Creative Commons Atribuição 3.0 não Adaptada.

Para ver uma cópia desta licença, visite http://creativecommons.org/licenses/by/3.0/deed.pt_BR. 